\title{
PAPER
}

\section{The shape of two-dimensional liquid bridges}

To cite this article: Paulo I C Teixeira and Miguel A C Teixeira 2020 J. Phys.: Condens. Matter 32034002

View the article online for updates and enhancements.

\section{IOP ebooks}

Bringing you innovative digital publishing with leading voices

to create your essential collection of books in STEM research.

Start exploring the collection - download the first chapter of every title for free. 


\title{
The shape of two-dimensional liquid bridges
}

\author{
Paulo I C Teixeira ${ }^{1,2}$ and Miguel A C Teixeira ${ }^{3} \oplus$ \\ 1 ISEL-Instituto Superior de Engenharia de Lisboa, Instituto Politécnico de Lisboa Rua Conselheiro \\ Emídio Navarro 1, 1959-007 Lisbon, Portugal \\ 2 Centro de Física Teórica e Computacional, Faculdade de Ciências da Universidade de Lisboa Campo \\ Grande, Edifício C8, 1749-016 Lisbon, Portugal \\ ${ }^{3}$ Department of Meteorology, University of Reading Earley Gate, PO Box 243, Reading RG6 6BB, \\ United Kingdom \\ E-mail: piteixeira@fc.ul.pt and m.a.teixeira@reading.ac.uk
}

Received 26 July 2019, revised 16 September 2019

Accepted for publication 27 September 2019

Published 23 October 2019

\begin{abstract}
We have studied a single vertical, two-dimensional liquid bridge spanning the gap between two flat, horizontal solid substrates of given wettabilities. For this simple geometry, the Young-Laplace equation can be solved (quasi-)analytically to yield the equilibrium bridge shape under gravity. We establish the range of gap widths (as described by a Bond number Bo) for which the liquid bridge can exist, for given contact angles at the top and bottom substrates $\left(\theta_{c}^{t}\right.$ and $\theta_{c}^{b}$, respectively). In particular, we find that the absolute maximum span of a liquid bridge is four capillary lengths, for $\theta_{c}^{b}=180^{\circ}$ and $\theta_{c}^{t}=0^{\circ}$; whereas for $\theta_{c}^{b}=0^{\circ}$ and $\theta_{c}^{t}=180^{\circ}$ no bridge can form, for any substrate separation. We also obtain the minimum value of the cross-sectional area of such a liquid bridge, as well as the conditions for the existence and positions of any necks or bulges and inflection points on its surface. This generalises our earlier work in which the gap was assumed to be spanned by a liquid film of zero thickness connecting two menisci at the bottom and top substrates.
\end{abstract}

Keywords: capillary bridges, Young-Laplace equation, surface tension, theory and modelling, foams and emulsions, soap films

(Some figures may appear in colour only in the online journal)

\section{Introduction}

In a recent paper [1] we investigated the equilibrium shapes, under gravity, of the two-dimensional (2D) Plateau borders along which a single vertical soap film contacts two flat, horizontal solid substrates of given wettabilities. For this simple geometry, the Young-Laplace equation can be solved (quasi-) analytically, and we showed that these Plateau borders, where most of a foam's liquid resides, can only exist if the values of the Bond number Bo and of the liquid contact angle $\theta_{c}$ lie within certain domains in $\left(\theta_{c}\right.$, Bo $)$ space: under these conditions the substrate is foam-philic. For values outside these domains, the substrate cannot support a soap film and it is foam-phobic. However we assumed-as is common and reasonable in dry foams - that the soap film has zero thickness, implying that the top and bottom Plateau borders are effectively de-coupled. If the film is not infinitesimally thin, what we have instead is a liquid bridge or capillary bridge. Liquid bridges are relevant in many contexts, such as sand art [2]; atomic-force microscopy in high-humidity environments [3]; soldering [4]; the testing of weakly-adhesive solid surfaces [5]; in lungs, where they may close small airways and impair gas exchange [6]; the wet adhesion of insects and tree frogs [7]; the feeding of shore birds [8]; the spontaneous filling of porous materials [9]; or as tools for contact angle measurements [10]. Liquid bridges may cause attraction or repulsion between the bodies they connect, which may be surfaces (flat or curved), particles, or other liquids [11].

Studies of liquid bridges in zero gravity go back more than 150 years to Delaunay [12], who solved the YoungLaplace equation for the surface-area minimising shapes of an axisymmetric bridge in zero gravity; these shapes were later 
classified by Plateau [13] and their stability investigated by Lord Rayleigh [14]. Most research to date has concentrated on this particular experimentally-relevant geometry, in either zero or non-zero gravity [15-36].

It should be noted that the presence of gravity complicates matters substantially, as it precludes an analytical solution for the bridge shape. Here we shall follow a different route and generalise our work on 2D Plateau borders [1] to consider a slab of liquid between two flat, unbounded horizontal substrates at which the contact angles are fixed. In the terminology introduced by Fortes [23] these are $\theta$-bridges, and perhaps the nearest experimental realisation of the slab geometry is a slit pore (see, e.g. [37] and references therein). This is of both fundamental and practical relevance, as it is now possible to fabricate substrates with specified wetting properties [38]. The key advantage of our approach is that it allows us expeditiously to discern the effect of gravity on bridge shapes and properties, which has been neglected in most previous work. At this stage, we do not explicitly examine the stability of our $2 \mathrm{D}$ bridges with respect to other shapes (e.g. axisymmetric) but, as we shall argue below, this does not substantially restrict the applicability of our results.

This paper is organised as follows: in section 2 we write down the Young-Laplace equation for the bounding surfaces of a 2D liquid bridge. This is then solved (quasi-)analytically, for arbitrary gravity and liquid contact angles at the bottom and top substrates. We next derive the ranges of parameters for which such liquid bridges may exist. Results for the bridge shapes and minimum cross-sectional areas are presented in section 3. Finally we summarise and conclude in section 4.

\section{Theory}

The Young-Laplace law for the 2D (i.e. slab-symmetric) surfaces bounding a liquid bridge between two flat horizontal substrates (see figure 1) can be written [39]:

$$
\left[1+\left(\frac{\mathrm{d} x}{\mathrm{~d} z}\right)^{2}\right]^{-3 / 2} \frac{\mathrm{d}^{2} x}{\mathrm{~d} z^{2}}=-\frac{\Delta p}{\gamma}
$$

where $z$ is height measured from the bottom substrate, $x$ is the distance measured horizontally from the plane of symmetry (the midplane of the 2D bridge), $\Delta p(z)$ is the pressure difference across the bridge surface at each height, and $\gamma$ is the surface tension of the liquid.

Our aim is to solve equation (1) for one of the surfaces bounding the bridge. Naturally, the other surface is mirrorsymmetric with respect to $x=0$. We define $\Delta p=p_{2}-p_{1}$, where $p_{2}$ is the pressure inside the bridge (i.e. within the liquid) and $p_{1}$ is the atmospheric pressure outside the bridge (assumed to be constant). If the bridge is in hydrostatic equilibrium, we have

$$
\Delta p=p_{2}-p_{1}=p_{20}-p_{1}-\rho g z
$$

where $p_{20}$ is the pressure inside the bridge at the bottom substrate $(z=0), g$ is the gravitational acceleration, and $\rho$ is the density of the liquid inside the bridge.

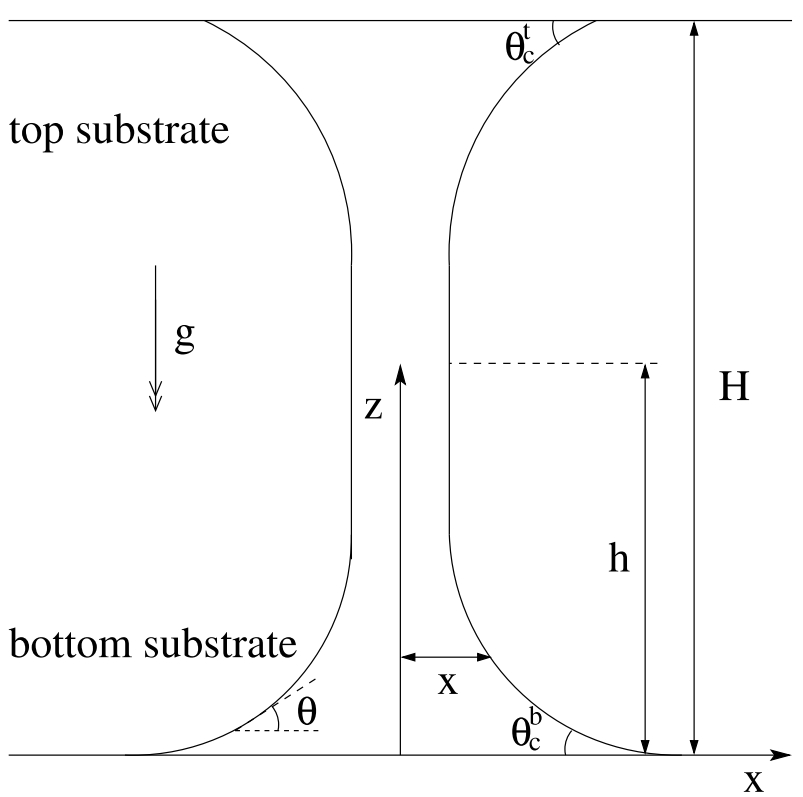

Figure 1. Sketch of a slab-symmetric liquid bridge spanning the gap between two flat horizontal substrates: $z$ is the height above the bottom substrate, $x$ is the distance from the midplane of the bridge (the $z$-axis) to the bridge border surface, $H$ is the substrate separation, $h$ is the position of the bridge neck (i.e. where its surface is vertical), $\theta$ is the bridge surface inclination, and $\theta_{c}^{t}$ and $\theta_{c}^{b}$ are the liquid contact angles at the top $(z=H)$ and bottom $(z=0)$ substrates, respectively. The gravitational acceleration is $g$.

Additionally, we introduce the convenient change of variables

$$
\frac{\mathrm{d} x}{\mathrm{~d} z}=-\cot \theta \quad \Rightarrow \quad \frac{\mathrm{d}^{2} x}{\mathrm{~d} z^{2}}=\frac{1}{\sin ^{2} \theta} \frac{\mathrm{d} \theta}{\mathrm{d} z}
$$

where $\theta$ is the inclination of the bridge surface (see figure 1), defined as the angle between the tangent to the bridge surface at point $(x, z)$ and the horizontal axis $(0 \leqslant \theta \leqslant \pi)$. Using equations (2) and (3), equation (1) becomes

$$
\sin \theta \frac{\mathrm{d} \theta}{\mathrm{d} z}=\frac{p_{1}-p_{20}}{\gamma}+\frac{\rho g z}{\gamma} .
$$

This equation can be straightforwardly solved for $\theta$, yielding

$$
\cos \theta(z)=\cos \theta_{c}^{b}-\frac{p_{1}-p_{20}}{\gamma} z-\frac{\rho g}{2 \gamma} z^{2}
$$

where the integration has been carried out from the base of the bridge, $z=0$, where $\theta=\theta_{c}^{b}$, to a generic height $z$. By definition, $\theta_{c}^{b}$ is the contact angle of the liquid with the underlying (bottom) solid substrate, and varies in the interval $0<\theta_{c}^{b}<\pi$. If equation (4) is instead integrated from $z=0$ to the top substrate, $z=H$, where $\theta=\pi-\theta_{c}^{t 4}$, this provides a definition for the pressure term on the right-hand side of equation (5), which allows us to eliminate this term:

$$
\frac{p_{1}-p_{20}}{\gamma}=\frac{1}{H}\left(\cos \theta_{c}^{b}+\cos \theta_{c}^{t}\right)-\frac{\rho g H}{2 \gamma} .
$$

${ }^{4}$ Recall that $\theta$ is the inclination angle measured relative to the horizontal axis $x$, so $\theta$ equals the physical contact angle, $\theta_{c}^{b}$, at the bottom substrate, but the supplementary contact angle, $\pi-\theta_{c}^{t}$, at the top substrate. 
Equation (5) can now be expressed entirely in terms of $z, H$, $\theta_{c}^{b}$ and $\theta_{c}^{t}$

$$
\cos \theta(z)=\cos \theta_{c}^{b}-\frac{1}{H}\left(\cos \theta_{c}^{b}+\cos \theta_{c}^{t}\right) z+\frac{\rho g z}{2 \gamma}(H-z)
$$

This equation can be written more simply if $z$ is made dimensionless by scaling it by $H$, the separation between top and bottom substrates, such that $z^{\prime}=z / H$, and a Bond number is defined as Bo $=\rho g H^{2} / \gamma$. In terms of these quantities, equation (7) can be rewritten as

$$
\cos \theta\left(z^{\prime}\right)=-\cos \theta_{c}^{t} z^{\prime}+\left(1-z^{\prime}\right)\left(\cos \theta_{c}^{b}+\frac{\mathrm{Bo}}{2} z^{\prime}\right) .
$$

To obtain $x$ as a function of $z$, we now go back to the definition of $\mathrm{d} x / \mathrm{d} z$. Further defining $x^{\prime}=x / H$, it follows that

$$
\frac{\mathrm{d} x^{\prime}}{\mathrm{d} z^{\prime}}=\frac{\mathrm{d} x}{\mathrm{~d} z}=-\cot \theta=-\frac{\cos \theta}{\sqrt{1-\cos ^{2} \theta}} .
$$

Using equation (8), equation (9) can be rewritten as

$$
\frac{\mathrm{d} x^{\prime}}{\mathrm{d} z^{\prime}}=-\frac{-\cos \theta_{c}^{t} z^{\prime}+\left(1-z^{\prime}\right)\left(\cos \theta_{c}^{b}+\frac{\mathrm{Bo}}{2} z^{\prime}\right)}{\left\{1-\left[-\cos \theta_{c}^{t} z^{\prime}+\left(1-z^{\prime}\right)\left(\cos \theta_{c}^{b}+\frac{\mathrm{Bo}}{2} z^{\prime}\right)\right]^{2}\right\}^{1 / 2}}
$$

which can be integrated between $z^{\prime}=0$ and a generic $z^{\prime}$, yielding

$$
x^{\prime}\left(z^{\prime}\right)=x^{\prime}(0)-\int_{0}^{z^{\prime}} \frac{-\cos \theta_{c}^{t} z^{\prime \prime}+\left(1-z^{\prime \prime}\right)\left(\cos \theta_{c}^{b}+\frac{\mathrm{Bo}}{2} z^{\prime \prime}\right)}{\left\{1-\left[-\cos \theta_{c}^{t} z^{\prime \prime}+\left(1-z^{\prime \prime}\right)\left(\cos \theta_{c}^{b}+\frac{\mathrm{Bo}}{2} z^{\prime \prime}\right)\right]^{2}\right\}^{1 / 2}} \mathrm{~d} z^{\prime \prime} .
$$

This equation gives the shape of the right-hand surface $\left(x^{\prime}\left(z^{\prime}\right) \geqslant 0\right)$ bounding the bridge between the bottom $\left(z^{\prime}=0\right)$ and top $\left(z^{\prime}=1\right)$ substrates. In zero gravity $(\mathrm{Bo}=0)$ the integral in equation (11) can be performed analytically, with the result

$x^{\prime}\left(z^{\prime}\right)=x^{\prime}(0)+\frac{\sin \theta_{c}^{b}-\left\{1-\left[\cos \theta_{c}^{b}-\left(\cos \theta_{c}^{b}+\cos \theta_{c}^{t}\right) z^{\prime}\right]^{2}\right\}^{1 / 2}}{\cos \theta_{c}^{b}+\cos \theta_{c}^{t}}$

which, as expected, is an arc of circle, meeting the bottom and top substrates at angles $\theta_{c}^{b}$ and $\theta_{c}^{t}$, respectively.

Another relevant quantity is the cross-sectional area of the liquid bridge. This is defined as

$$
A=2 \int_{0}^{H} x \mathrm{~d} z=2[z x]_{0}^{H}-2 \int_{0}^{H} z \frac{\mathrm{d} x}{\mathrm{~d} z} \mathrm{~d} z=2 H x(H)-2 \int_{0}^{H} z \frac{\mathrm{d} x}{\mathrm{~d} z} \mathrm{~d} z
$$

where the second equality follows from integrating by parts. The factor of 2 in equation (13) accounts for the fact that the bridge surfaces are symmetric about $x=0$. Defining a dimensionless area as $A^{\prime}=A / H^{2}$, this is given, from equation (13), by

$$
A^{\prime}=2 x^{\prime}(1)-2 \int_{0}^{1} z^{\prime} \frac{\mathrm{d} x^{\prime}}{\mathrm{d} z^{\prime}} \mathrm{d} z^{\prime} .
$$

Using equation (10), equation (14) can be written explicitly as

$$
A^{\prime}=2 x^{\prime}(1)+2 \int_{0}^{1} \frac{-\cos \theta_{c}^{t} z^{2}+\left(1-z^{\prime}\right)\left(\cos \theta_{c}^{b}+\frac{\mathrm{Bo}}{2} z^{\prime}\right) z^{\prime}}{\left\{1-\left[-\cos \theta_{c}^{t} z^{\prime}+\left(1-z^{\prime}\right)^{2}\left(\cos \theta_{c}^{b}+\frac{\mathrm{Bo}}{2} z^{\prime}\right)\right]^{2}\right\}^{1 / 2}} \mathrm{~d} z^{\prime} .
$$

It is also easy to locate the position(s) $z=h$ where the bridge surfaces are vertical (perpendicular to the substrates), which we shall call 'necks' if the bridge surface is concave there (i.e. $\mathrm{d}^{2} x / \mathrm{d} z^{2}>0$ ) and 'bulges' if it is convex (i.e. $\left.\mathrm{d}^{2} x / \mathrm{d} z^{2}<0\right)$. This follows from setting $\cos \theta(z=h)=0$ in equation (8), whence, in reduced units where $h^{\prime}=h / H$ :

Bo $h^{\prime 2}-\left[\right.$ Bo $\left.-2\left(\cos \theta_{c}^{b}+\cos \theta_{c}^{t}\right)\right] h^{\prime}-2 \cos \theta_{c}^{b}=0$

which can be straightforwardly solved to yield

$h^{\prime}=\frac{\mathrm{Bo}-2\left(\cos \theta_{c}^{b}+\cos \theta_{c}^{t}\right) \pm \sqrt{\mathrm{Bo}^{2}-4\left(\cos \theta_{c}^{t}-\cos \theta_{c}^{b}\right) \mathrm{Bo}+4\left(\cos \theta_{c}^{t}+\cos \theta_{c}^{b}\right)^{2}}}{2 \mathrm{Bo}}$

This can have either no real roots, or one or two real roots, in the physically-meaningful range $0 \leqslant h^{\prime} \leqslant 1$. In zero gravity $(\mathrm{Bo}=0)$, equation $(16)$ simplifies to

$$
h^{\prime}=\frac{\cos \theta_{c}^{b}}{\cos \theta_{c}^{b}+\cos \theta_{c}^{t}} .
$$

If, in addition, the contact angles are the same at either substrate $\left(\theta_{c}^{b}=\theta_{c}^{t}\right)$, we obtain $h=H / 2$, as would be expected on physical grounds.

Finally, it is relevant to know if inflection points, i.e. points of local zero curvature where $\mathrm{d}^{2} x / \mathrm{d} z^{2}=0$, exist on the surfaces bounding the liquid bridge. Their location $\tilde{h}$ is obtained from equation (3), by imposing that $\mathrm{d} \theta / \mathrm{d} z=0$, or equivalently that $\mathrm{d}(\cos \theta) / \mathrm{d} z=0$, which yields the following dimensionless height $\tilde{h}^{\prime}=\tilde{h} / H$ :

$$
\tilde{h}^{\prime}=\frac{1}{2}-\frac{\cos \theta_{c}^{b}+\cos \theta_{c}^{t}}{\text { Bo }} .
$$

This solution will only be physically meaningful if $0<\tilde{h}^{\prime}<1$. Clearly, in the absence of gravity $(\mathrm{Bo}=0)$ there are no inflection points. The conditions for the existence of necks/bulges given by equation (17), and of inflection points given by equation (19), will be discussed in section 3 .

We end this section by noting that $x^{\prime}(0)$, which equals half the bridge width at the bottom substrate, is not known $a$ priori. This is a consequence of the fact that we are treating the two bridge surfaces independently: $x^{\prime}(0)$ is thus determined by the (arbitrary) total amount of liquid in the bridge. If that amount is large enough, then the bridge will be stable, because the areas of its surfaces will necessarily be minimal for a given liquid volume. For definiteness and clarity of presentation, in what follows $x^{\prime}(0)$ is fixed so that $x^{\prime}\left(z^{\prime}\right) \geqslant 0$ for all $0 \leqslant z^{\prime} \leqslant 1$, as follows:

- If the bridge has a single neck at some $z^{\prime}=h^{\prime}$ $\left(0 \leqslant h^{\prime} \leqslant 1\right)$, we find $x^{\prime}(0)$ from equation (11) by requiring that $x^{\prime}\left(h^{\prime}\right)=0$.

- If the bridge has a bulge and a neck, at $z^{\prime}=h_{1}^{\prime}$ and $z^{\prime}=h_{2}^{\prime}$ $\left(0 \leqslant h_{1}^{\prime}<h_{2}^{\prime} \leqslant 1\right)$, we find $x^{\prime}(0)$ from equation (11) by requiring that $x_{i}^{\prime}=0$, where $x_{i}^{\prime}=\min \left[x^{\prime}\left(h_{1}^{\prime}\right), x^{\prime}\left(h_{2}^{\prime}\right)\right]$ (which corresponds to the neck).

- If the bridge has no necks/bulges, we first set $x^{\prime}(0)=0$ and solve equation (11). Then if $x^{\prime}(1) \geqslant 0$, we keep $x^{\prime}(0)=0$; otherwise if $x^{\prime}(1)<0$, we find $x^{\prime}(0)$ by translating the bridge along the $x$-axis so that $x^{\prime}(1)=0$. 
The above procedure ensures that our bridges have the minimum cross-sectional area, as their two bounding surfaces touch. This will be the minimum amount of liquid needed to make a bridge between two substrates a distance $H$ apart, for given $\theta_{c}^{b}$ and $\theta_{c}^{t}$. In and of themselves, such bridges are most likely to be unstable, so one alternative interpretation of the minimum cross-sectional area is as an excess quantity: the minimum amount of liquid that is needed for a bridge to form between two substrates in addition to that contained in the 'bulk' of the bridge - a central slab of liquid of arbitrary thickness, with straight sides perpendicular to said substrates. We end this section by noting that all other calculated quantities - the range of Bond numbers for which a bridge can exist and whether it has bulges/necks and/or inflection points on its surface - do not depend on bridge width, i.e. on the amount of liquid it contains, and so should remain generally valid.

\section{Results and discussion}

Equations (11) and (15) do not yield physically meaningful results for all values of Bo, $\theta_{c}^{b}$ and $\theta_{c}^{t}$. We next discuss the nontrivial conditions defining their domains of validity.

Our starting point is equation (8). Physically meaningful solutions will only exist if $-1 \leqslant \cos \theta \leqslant 1$, whence we must have

$-1 \leqslant-\cos \theta_{c}^{t} z^{\prime}+\left(1-z^{\prime}\right)\left(\cos \theta_{c}^{b}+\frac{\mathrm{Bo}}{2} z^{\prime}\right) \leqslant 1$.

The lower and upper bounds of equation (20) both correspond to $\sin \theta=0$, which causes singularities in the integrals that give $x^{\prime}\left(z^{\prime}\right)$ in equation (11) and $A^{\prime}$ in equation (15).

First of all, it should be noted that, in order to obtain meaningful liquid bridge solutions, these must be valid for every $z^{\prime}$ between 0 and 1 . It can be shown by differentiating equation (8) that $\cos \theta\left(z^{\prime}\right)$ varies monotonically with $z^{\prime}$, and thus its extrema occur either at $z^{\prime}=0$ or $z^{\prime}=1$, if Bo $<2\left|\cos \theta_{c}^{b}+\cos \theta_{c}^{t}\right|$. In this case, equation (20) is automatically satisfied because it is satisfied for $z^{\prime}=0$ and $z^{\prime}=1$ by construction of the solution. Hence what needs to be ascertained for every $z^{\prime}$ is whether equation (20) holds when $\mathrm{Bo} \geqslant 2\left|\cos \theta_{c}^{b}+\cos \theta_{c}^{t}\right|$. It is worth remarking that this is also the condition for the existence of inflection points on the bridge surface, since it follows from equation (19) by requiring that $0 \leqslant \tilde{h}^{\prime} \leqslant 1$.

Consider first the left-hand inequality in equation (20), which can be alternatively expressed as

Bo $z^{\prime 2}-\left[\right.$ Bo $\left.-2\left(\cos \theta_{c}^{b}+\cos \theta_{c}^{t}\right)\right] z^{\prime}-2\left(1+\cos \theta_{c}^{b}\right) \leqslant 0$.

This will be satisfied if

$$
\begin{aligned}
z^{\prime} & \geqslant \frac{\mathrm{Bo}-2\left(\cos \theta_{c}^{b}+\cos \theta_{c}^{t}\right)-\sqrt{\left[\mathrm{Bo}-2\left(\cos \theta_{c}^{b}+\cos \theta_{c}^{t}\right)\right]^{2}+8 \mathrm{Bo}\left(1+\cos \theta_{c}^{b}\right)}}{2 \mathrm{Bo}} \\
\text { and } \quad z^{\prime} & \leqslant \frac{\mathrm{Bo}-2\left(\cos \theta_{c}^{b}+\cos \theta_{c}^{t}\right)+\sqrt{\left[\mathrm{Bo}-2\left(\cos \theta_{c}^{b}+\cos \theta_{c}^{t}\right)\right]^{2}+8 \mathrm{Bo}\left(1+\cos \theta_{c}^{b}\right)}}{2 \mathrm{Bo}}
\end{aligned}
$$

provided that the discriminant of equation (21) (i.e. the expression under the square roots in equation (22)) is non-negative, which is always true. It can be shown by explicit calculation

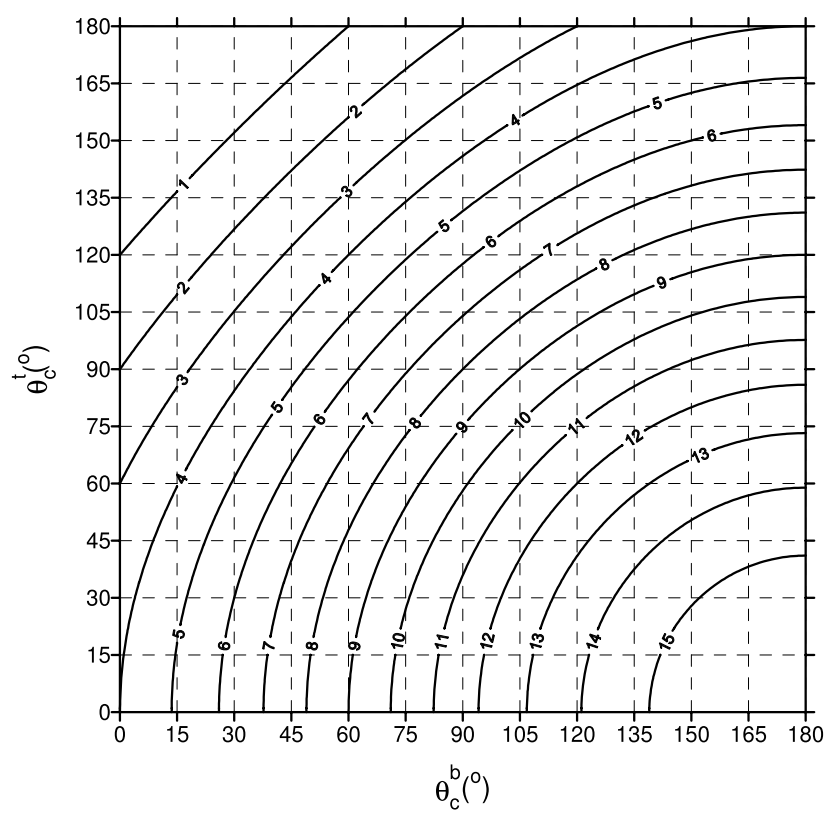

Figure 2. Level curves for the maximum Bond number for which a liquid bridge can exist, for given contact angles at the bottom and top substrates.

that the expression on the right-hand side of the first inequality in equation (22) is never $>0$, and (less obviously) that the expression on the right-hand side of the second inequality in equation (22) is never $<1$, which necessarily implies that equation (22) is satisfied for all $0<z^{\prime}<1$, and therefore that equation (21) is itself satisfied for all Bo, $\theta_{c}^{b}$ and $\theta_{c}^{t}$.

We now turn to the right-hand inequality in equation (20), which can be alternatively expressed as

$$
\text { Bo } z^{\prime 2}-\left[\text { Bo }-2\left(\cos \theta_{c}^{b}+\cos \theta_{c}^{t}\right)\right] z^{\prime}+2\left(1-\cos \theta_{c}^{b}\right) \geqslant 0 \text {. }
$$

This will be satisfied if

$$
\begin{aligned}
z^{\prime} & \leqslant \frac{\mathrm{Bo}-2\left(\cos \theta_{c}^{b}+\cos \theta_{c}^{t}\right)-\sqrt{\left[\mathrm{Bo}-2\left(\cos \theta_{c}^{b}+\cos \theta_{c}^{t}\right)\right]^{2}-8 \mathrm{Bo}\left(1-\cos \theta_{c}^{b}\right)}}{2 \mathrm{Bo}} \\
\text { or } z^{\prime} & \geqslant \frac{\mathrm{Bo}-2\left(\cos \theta_{c}^{b}+\cos \theta_{c}^{t}\right)+\sqrt{\left[\mathrm{Bo}-2\left(\cos \theta_{c}^{b}+\cos \theta_{c}^{t}\right)\right]^{2}-8 \mathrm{Bo}\left(1-\cos \theta_{c}^{b}\right)}}{2 \mathrm{Bo}}
\end{aligned}
$$

provided that the discriminant of equation (23) (i.e. the expression under the square roots in equation (24)) is non-negative (otherwise equation (23) will be satisfied by default). For Bo $\geqslant 2\left|\cos \theta_{c}^{b}+\cos \theta_{c}^{t}\right|$, as assumed above, it can be shown that equation (24) will only be satisfied for some $0<z^{\prime}<1$. Therefore, the condition that must be met for equation (23) to be satisfied for all $z^{\prime}$ is that its discriminant must be negative, which from equation (24) can be expressed as

$$
\mathrm{Bo}^{2}+4\left(\cos \theta_{c}^{b}-\cos \theta_{c}^{t}-2\right) \mathrm{Bo}+4\left(\cos \theta_{c}^{b}+\cos \theta_{c}^{t}\right)^{2}<0 .
$$

This will hold as long as

$$
\begin{aligned}
\text { Bo }>2\left(2-\cos \theta_{c}^{b}+\cos \theta_{c}^{t}\right)-4 \sqrt{\left(1-\cos \theta_{c}^{b}\right)\left(1+\cos \theta_{c}^{t}\right)} \\
\text { and } \quad \text { Bo }<2\left(2-\cos \theta_{c}^{b}+\cos \theta_{c}^{t}\right)+4 \sqrt{\left(1-\cos \theta_{c}^{b}\right)\left(1+\cos \theta_{c}^{t}\right)}
\end{aligned}
$$




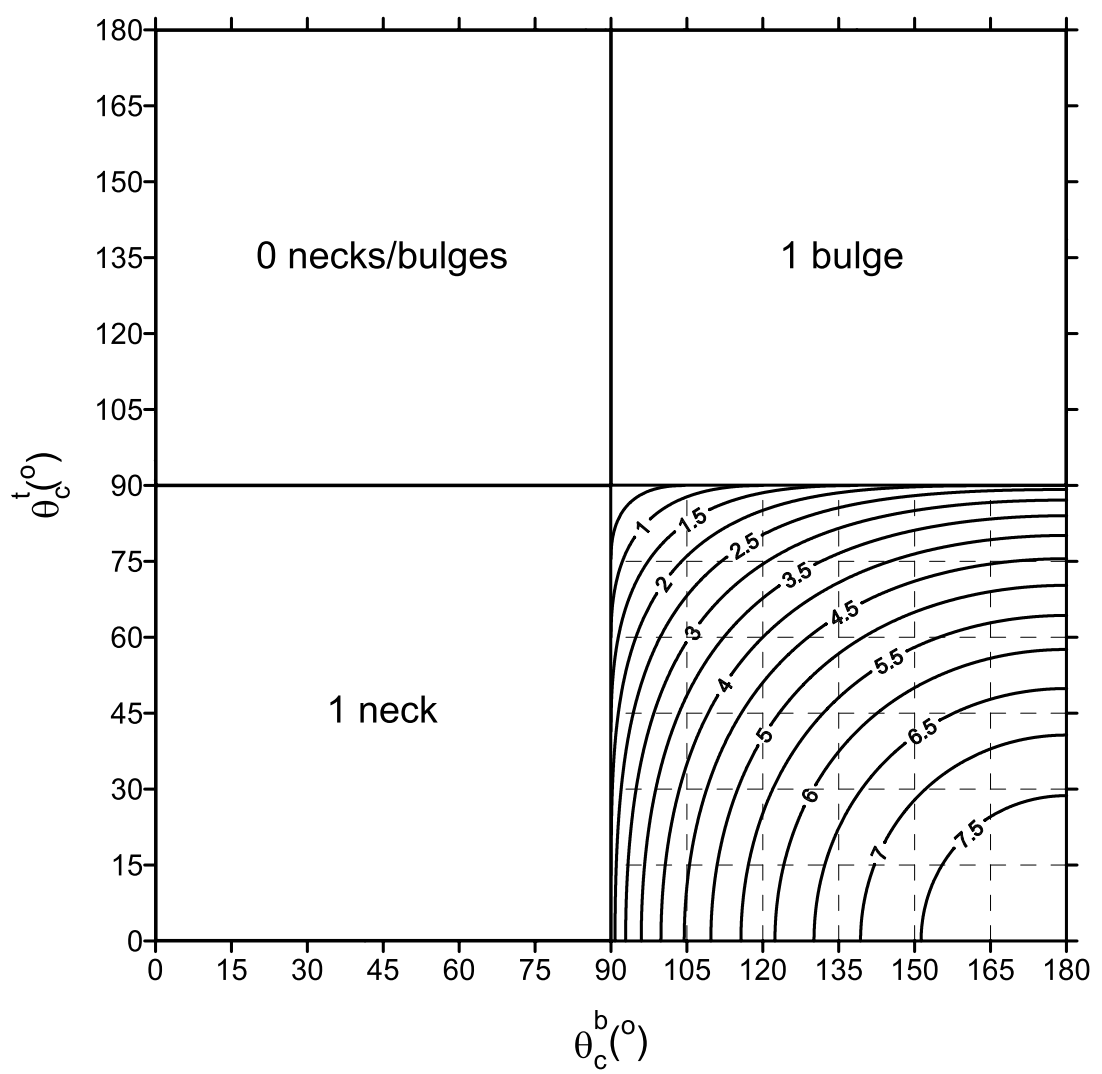

Figure 3. Regime diagram showing the number of necks/bulges on a liquid bridge for given contact angles at the bottom and top substrates. Note that for $0^{\circ}<\theta_{c}^{b}<90^{\circ}$ and $90^{\circ}<\theta_{c}^{t}<180^{\circ}$ there are no necks or bulges. For $0^{\circ}<\theta_{c}^{b}<90^{\circ}$ and $0^{\circ}<\theta_{c}^{t}<90^{\circ}$ there is one neck, and for $90^{\circ}<\theta_{c}^{b}<180^{\circ}$ and $90^{\circ}<\theta_{c}^{t}<180^{\circ}$ there is one bulge. The curves in the quadrant where $90^{\circ}<\theta_{c}^{b}<180^{\circ}$ and $0^{\circ}<\theta_{c}^{t}<90^{\circ}$ are the loci of bifurcation points: below the value of Bo labelling each curve there are no necks or bulges, at that value there is a single 'degenerate' neck/bulge (which coincides with an inflection point), and above that value there is one neck and one bulge.

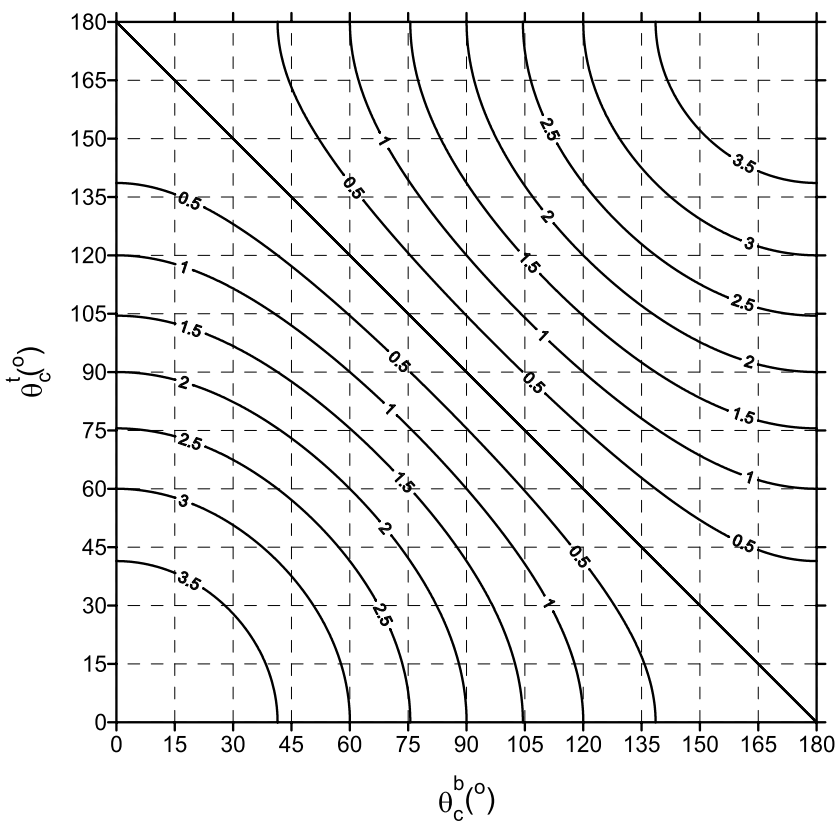

Figure 4. Level curves for the minimum Bond number for which a liquid bridge has an inflection point, for given contact angles at the bottom and top substrates. where the discriminant of equation (25) (i.e. 64 times the expression under the square roots in equation (26)) is clearly always non-negative. Now it can be shown by explicit calculation that when $\mathrm{Bo} \geqslant 2\left|\cos \theta_{c}^{b}+\cos \theta_{c}^{t}\right|$ the first inequality in equation (26) is always satisfied, hence the larger root provides an upper bound for Bo, for given $\left(\theta_{c}^{b}, \theta_{c}^{t}\right)$ (see figure 2):

$$
\text { Bo }<2\left(2-\cos \theta_{c}^{b}+\cos \theta_{c}^{t}\right)+4 \sqrt{\left(1-\cos \theta_{c}^{b}\right)\left(1+\cos \theta_{c}^{t}\right)} \text {. }
$$

Recalling the definition of Bo, this means that, for a given fluid in contact with a given pair of substrates, there is a maximum substrate separation beyond which a liquid bridge cannot span the gap between the two substrates: the bridge collapses under its own weight. In particular, the absolute maximum span of a liquid bridge is four capillary lengths: this is attained when the upper bound for the Bond number is greatest, $\mathrm{Bo}=16$, for $\theta_{c}^{b}=180^{\circ}$ and $\theta_{c}^{t}=0^{\circ}$ (see figure 2). By contrast, for $\theta_{c}^{b}=0^{\circ}$ and $\theta_{c}^{t}=180^{\circ}$, no bridge can form, for any substrate separation, because the upper bound for the Bond number is then Bo $=0$. Remarkably, unlike for axisymmetric bridges [40, 41], the maximum surface separation does not depend on bridge volume. 

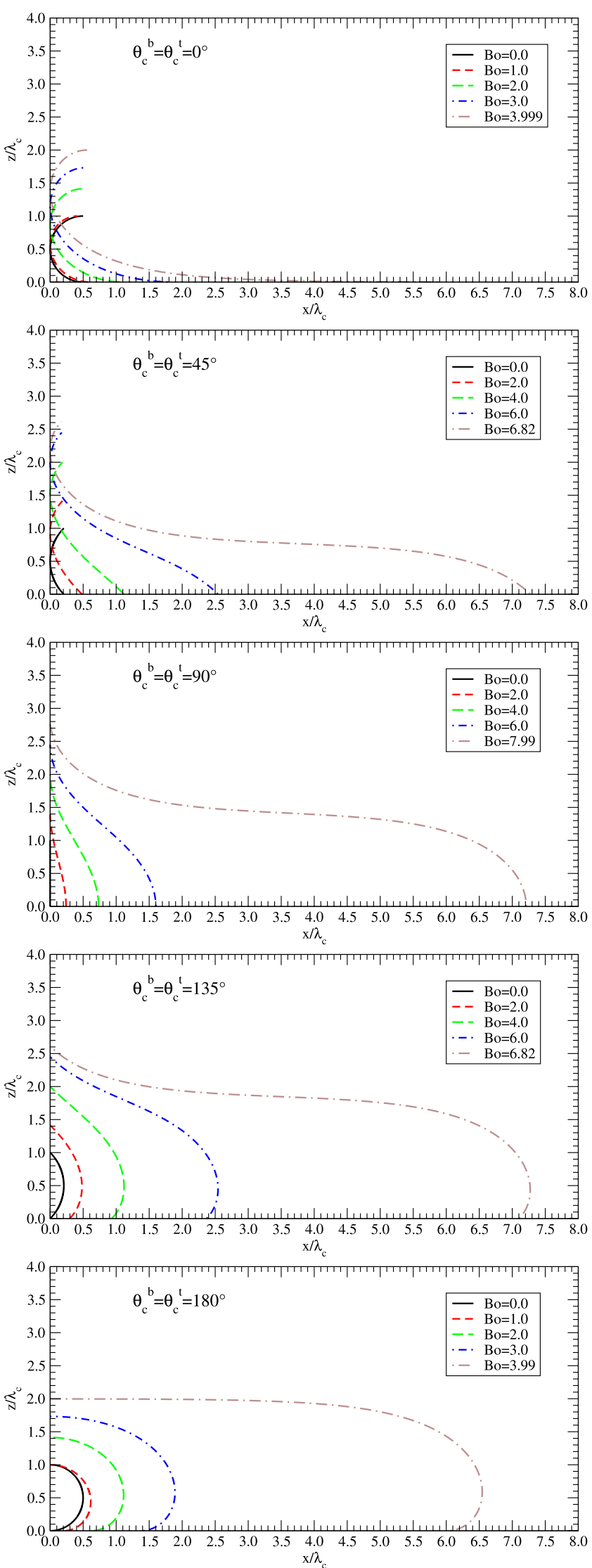

Figure 5. Shapes of liquid bridges between identical substrates. Lengths are in units of $\lambda_{c}$, the capillary length of the liquid. From top to bottom: $\theta_{c}^{b}=\theta_{c}^{t}=0^{\circ}, 45^{\circ}, 90^{\circ}, 135^{\circ}$ and $180^{\circ}$.

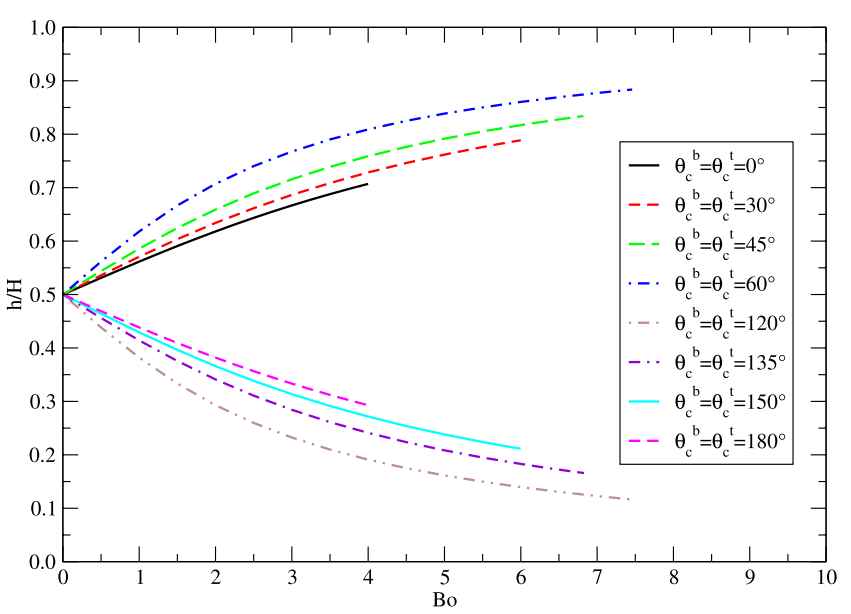

Figure 6. Scaled position of bridge neck/bulge $h^{\prime}=h / H$ versus Bo for identical substrates $\left(\theta_{c}^{b}=\theta_{c}^{t}\right)$. For $\theta_{c}^{b}=\theta_{c}^{t}=90^{\circ}$ the whole bridge surface is vertical if $\mathrm{Bo}=0$; if $\mathrm{Bo} \neq 0$ the necks/bulges are at the bottom and top substrates $\left(h_{1}^{\prime}=0, h_{2}^{\prime}=1\right)$, so this case is not shown.

We now systematically derive and discuss the conditions for the existence of points where the bridge surfaces are vertical, i.e. of necks and bulges as defined in section 2 .

From equation (17), for two necks/bulges to exist, the discriminant under the square root must be non-negative, and both solutions for $h^{\prime}$ must lie in the interval $0<h^{\prime}<1$. Actually, for topological reasons, this situation must always correspond to one neck and one bulge (it makes no sense to simultaneously have two necks or two bulges). Additionally, since the pressure must decrease upwards, the bridge curvature must become more concave higher up, which means that the neck must always lie above the bulge. If $0<h^{\prime}<1$ but the discriminant vanishes, or alternatively if the discriminant is positive but only one of the solutions given by equation (17) satisfies $0<h^{\prime}<1$, there will be only one neck or bulge. In all other cases, including (but not limited to) those in which the discriminant is negative, no necks or bulges can exist. A more extended analysis (see the appendix for details) leads to the following conditions:

- There is one neck and one bulge if $\cos \theta_{c}^{b}<0$ (i.e. $90^{\circ}<\theta_{c}^{b}<180^{\circ}$ ), $\cos \theta_{c}^{t}>0$ (i.e. $0^{\circ}<\theta_{c}^{t}<90^{\circ}$ ) and Bo $>2\left(\cos \theta_{c}^{t}-\cos \theta_{c}^{b}\right)+4 \sqrt{-\cos \theta_{c}^{b} \cos \theta_{c}^{t}}$.

- There is one neck if $\cos \theta_{c}^{b}>0$ (i.e. $0^{\circ}<\theta_{c}^{b}<90^{\circ}$ ) and $\cos \theta_{c}^{t}>0$ (i.e. $0^{\circ}<\theta_{c}^{t}<90^{\circ}$ ), since the bridge is concave; there is one bulge if $\cos \theta_{c}^{b}<0$ (i.e. $90^{\circ}<\theta_{c}^{b}<180^{\circ}$ ) and $\cos \theta_{c}^{t}<0$ (i.e. $90^{\circ}<\theta_{c}^{t}<180^{\circ}$ ), since the bridge is convex; and there is a 'degenerate' neck/bulge coinciding with an inflection point (intermediate between the shapes with no necks/bulges and those with one neck and one bulge) if $\cos \theta_{c}^{b}<0$ (i.e. $90^{\circ}<\theta_{c}^{b}<180^{\circ}$ ) and $\cos \theta_{c}^{t}>0$ (i.e. $0^{\circ}<\theta_{c}^{t}<90^{\circ}$ ) and $\mathrm{Bo}=2\left(\cos \theta_{c}^{t}-\cos \theta_{c}^{b}\right)+4 \sqrt{-\cos \theta_{c}^{b} \cos \theta_{c}}$.

- There are no necks or bulges if $\cos \theta_{c}^{b}>0$ (i.e. $0^{\circ}<\theta_{c}^{b}<90^{\circ}$ ) and $\cos \theta_{c}^{t}<0$ (i.e. $90^{\circ}<\theta_{c}^{t}<180^{\circ}$ ); or if $\cos \theta_{c}^{b}<0$ (i.e. $90^{\circ}<\theta_{c}^{b}<180^{\circ}$ ) and $\cos \theta_{c}^{t}>0$ (i.e. $\left.0^{\circ}<\theta_{c}^{t}<90^{\circ}\right)$ and $\mathrm{Bo}<2\left(\cos \theta_{c}^{t}-\cos \theta_{c}^{b}\right)+4 \sqrt{-\cos \theta_{c}^{b} \cos \theta_{c}^{t}}$. 
(a)

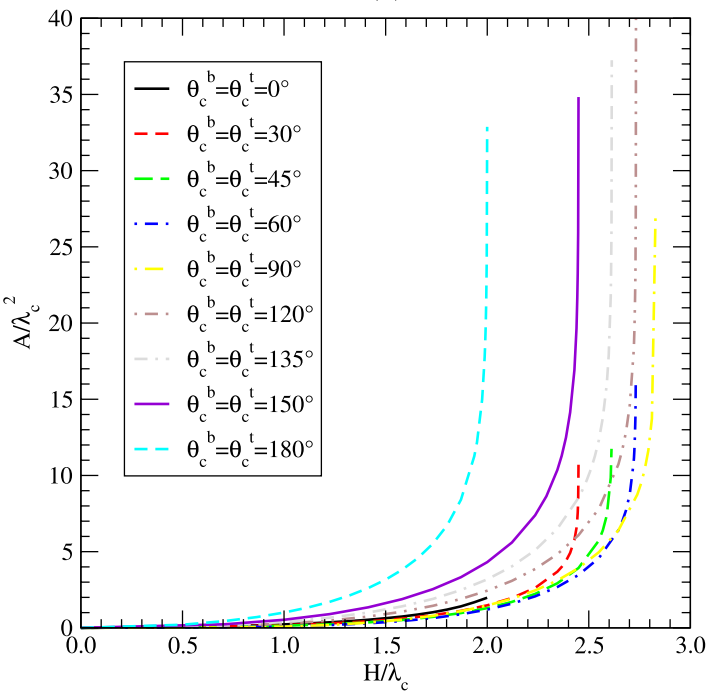

(b)

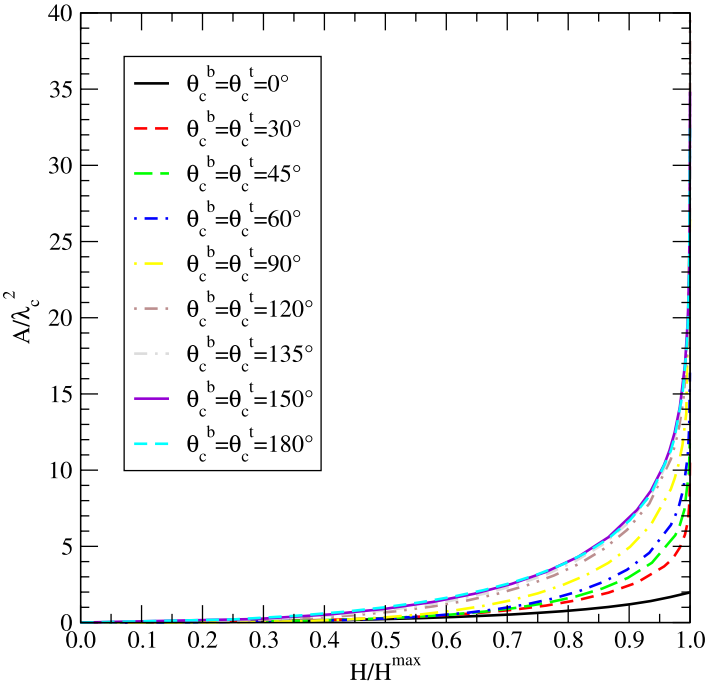

Figure 7. Dimensionless minimum cross-sectional area $A / \lambda_{c}^{2}$ of liquid bridge between identical substrates $\left(\theta_{c}^{b}=\theta_{c}^{t}\right)$ versus (a) substrate separation in units of $\lambda_{c}$ (which equals $\mathrm{Bo}^{1 / 2}$ ); (b) substrate separation scaled by its maximum value. The areas of all bridges with $\theta_{c}^{b}=\theta_{c}^{t} \neq 0$ diverge at the maximum substrate separation.

These results are summarised in figure 3. For reference, figure 4 plots the minimum Bo for which an inflection point can exist, which is just $\mathrm{Bo}=2\left|\cos \theta_{c}^{b}+\cos \theta_{c}^{t}\right|$. Note that both the values of Bo in the bottom-right quadrant of figure 3 and those in figure 4 are lower than those in figure 2, which means that bridges with two necks/bulges and with an inflection point are both realisable, although the limiting Bo in figures 2 and 4 coincide for $\theta_{c}^{b}=0^{\circ}$ and $\theta_{c}^{t}=0^{\circ}, \theta_{c}^{b}=0^{\circ}$ and $\theta_{c}^{t}=180^{\circ}$, and $\theta_{c}^{b}=180^{\circ}$ and $\theta_{c}^{t}=180^{\circ}$. Additionally, the limiting Bo in figure 4 are lower than those in figure 3 , which means that it is easier for bridges to have inflection points than one neck and one bulge. Examples of liquid bridges presented below will be interpreted in the light of these results.

In figure 5 we plot the shapes of liquid bridges between identical substrates, i.e. $\theta_{c}^{b}=\theta_{c}^{t}$. Recall that these are all minimum cross-sectional area bridges, as explained at the end of section 2, and are all mirror-symmetric with respect to $x=0$, so we only show one half of each. As Bo is increased from zero to its maximum value, the bridges become more and more top-bottom asymmmetric, or 'haunched', as gravity tends to pull down their liquid content. The two top panels are for contact angles in the bottom-left quadrant of figure 3, the two bottom panels are for contact angles in the top-right quadrant of figure 3 , and the middle panel is for contact angles at the centre of figure 3. It follows that these bridges all have only one neck or one bulge: for $\mathrm{Bo}=0$ (no gravity) the neck/bulge is located at $h=H / 2$ as we saw earlier; then as Bo increases, the neck position moves towards the top substrate if $\theta_{c}^{b}=\theta_{c}^{t}<90^{\circ}$, and the bulge position moves towards the bottom substrate if $\theta_{c}^{b}=\theta_{c}^{t}>90^{\circ}$. From top to bottom in figure 5 , inflection points are expected to exist for Bo $>4,2 \sqrt{2}, 0,2 \sqrt{2}, 4$, respectively (see figure 4 ). This is confirmed by the fact that none of the bridges in the top and bottom panels of figure 5 has any inflection points, that the middle-panel bridges all have one inflection point, and that the bridges in the second and fourth panels only have inflection points above a certain Bo (consistent with the thresholds quoted above). For all non-zero contact angles, the inflection point on the bridges migrates upwards from the bottom substrate as either Bo or $\theta_{c}^{b}=\theta_{c}^{t}$ are increased from zero, which is consistent with equation (19). The behaviour of necks/bulges, calculated using equation (17), is shown in figure 6: note the symmetry between contact angles $<90^{\circ}$ and $>90^{\circ}$. Figure 7 displays the minimum cross-sectional areas for bridges between identical substrates. These areas appear to diverge as Bo approaches its upper bound, except when $\theta_{c}^{b}=\theta_{c}^{t}=0^{\circ}$, as can be seen in figure 7(a). In this case, equation (15) for the bridge cross-sectional area simplifies, for the corresponding maximum Bond number $\mathrm{Bo}=4$, to

$$
A^{\prime}=2 x^{\prime}(1)+2 \int_{0}^{1} \frac{z^{\prime}\left(1-2 z^{\prime 2}\right)}{\left[1-\left(1-2 z^{\prime 2}\right)^{2}\right]^{1 / 2}} \mathrm{~d} z^{\prime}
$$

where the integral can be performed analytically and is seen to vanish. This means that $A^{\prime}=2 x^{\prime}(1)$, or if both $A$ and $x(H)$ are measured in units of capillarity length $\lambda_{c}=[\gamma /(\rho g)]^{1 / 2}$ instead of $H$ (as done in the figures),

$$
\frac{A}{\lambda_{c}^{2}}=4 \frac{x(H)}{\lambda_{c}}
$$

where we have used the fact that $\mathrm{Bo}=4$. This relationship can be confirmed by comparing the top panel of figure 5 with figure 7(a).

In figures 8 and 9 we plot the shapes of liquid bridges between hybrid substrates, i.e. $\theta_{c}^{b} \neq \theta_{c}^{t}$. Again, these are of minimum cross-sectional area. Now the bridges are always top-bottom asymmetric, even for $\mathrm{Bo}=0$, and may exhibit zero, one or two necks/bulges. For the bridges shown in the top and bottom panels of figure $8, \theta_{c}^{b}$ and $\theta_{c}^{t}$ lie in the bottomleft and top-right quadrants of figure 3 , respectively, so there is only one neck/bulge. In the second panel, $\theta_{c}^{b}$ and $\theta_{c}^{t}$ lie on 

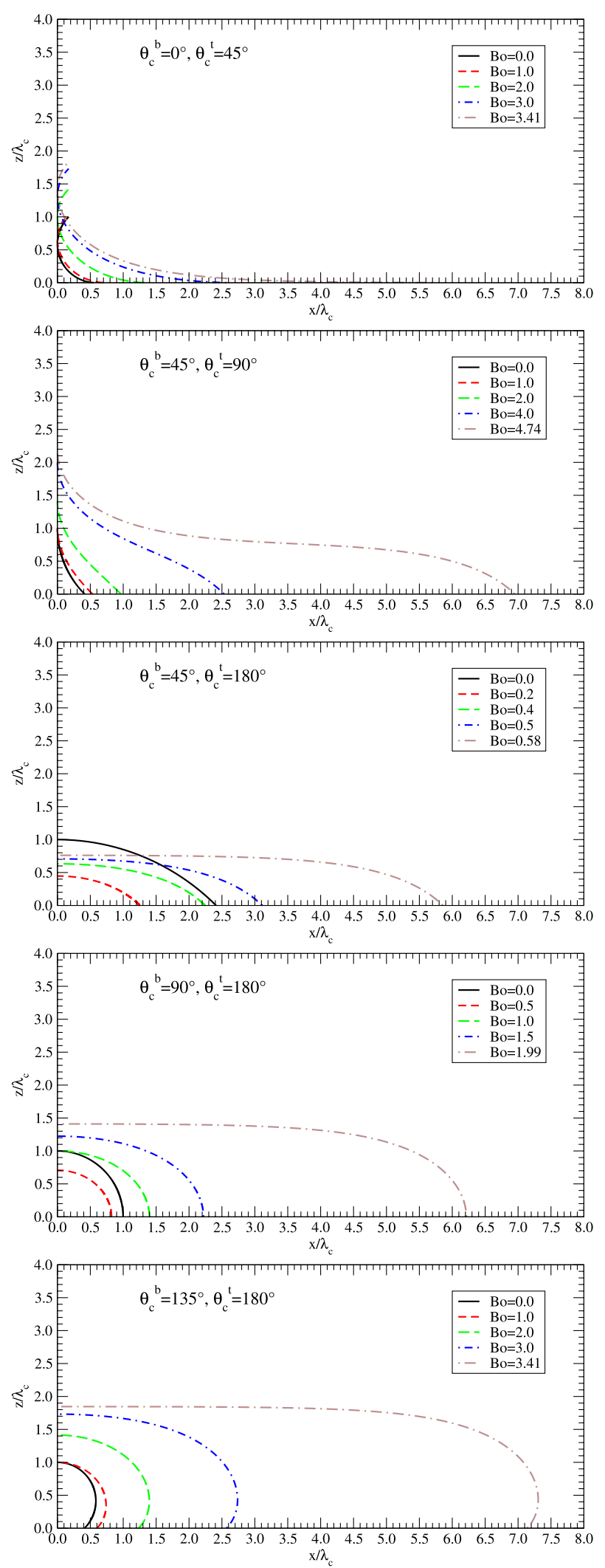

Figure 8. Shapes of liquid bridges between hybrid substrates $\left(\theta_{c}^{b}<\theta_{c}^{t}\right)$. Lengths are in units of $\lambda_{c}$, the capillary length of the liquid. From top to bottom: $\theta_{c}^{b}=0^{\circ}, \theta_{c}^{t}=45^{\circ}$; $\theta_{c}^{b}=45^{\circ}, \theta_{c}^{t}=90^{\circ} ; \theta_{c}^{b}=45^{\circ}, \theta_{c}^{t}=180^{\circ} ; \theta_{c}^{b}=90^{\circ}, \theta_{c}^{t}=180^{\circ}$; and $\theta_{c}^{b}=135^{\circ}, \theta_{c}^{t}=180^{\circ}$.
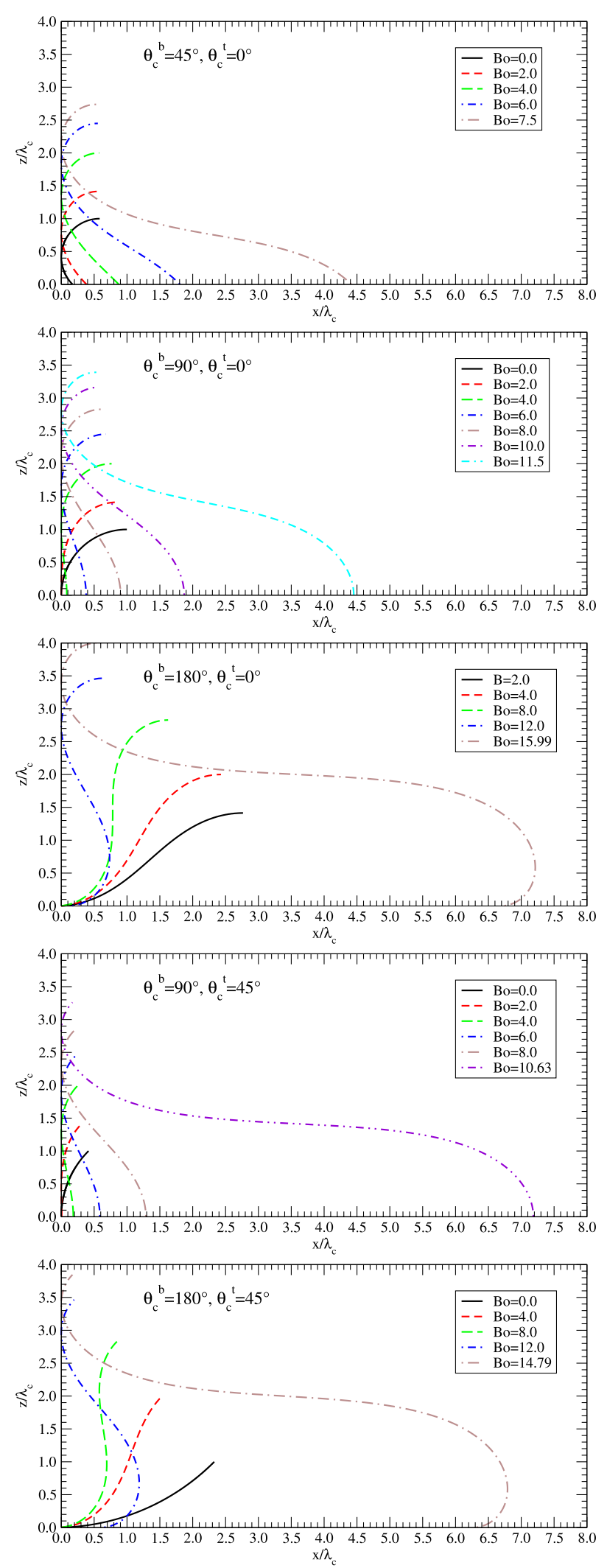

Figure 9. Shapes of liquid bridges between hybrid substrates $\left(\theta_{c}^{b}>\theta_{c}^{t}\right)$. Lengths are in units of $\lambda_{c}$, the capillary length of the liquid. From top to bottom: $\theta_{c}^{b}=45^{\circ}, \theta_{c}^{t}=0^{\circ} ; \theta_{c}^{b}=90^{\circ}, \theta_{c}^{t}=0^{\circ}$; $\theta_{c}^{b}=180^{\circ}, \theta_{c}^{t}=0^{\circ} ; \theta_{c}^{b}=90^{\circ}, \theta_{c}^{t}=45^{\circ}$; and $\theta_{c}^{b}=180^{\circ}, \theta_{c}^{t}=45^{\circ}$. 
(a)

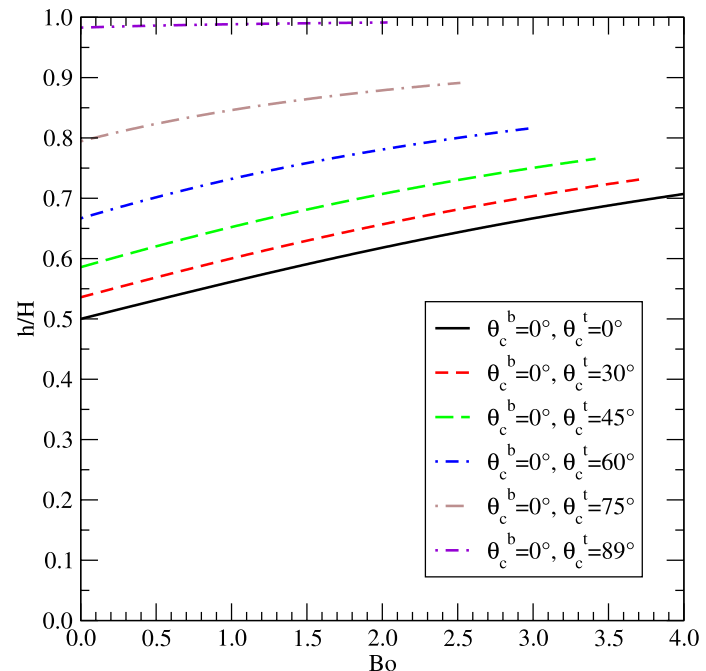

(b)

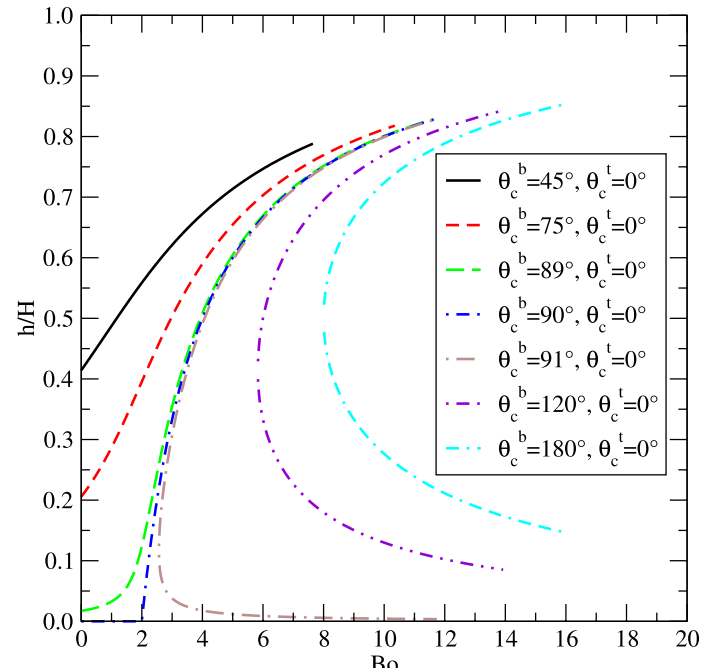

Figure 10. Scaled position of bridge necks/bulges $h^{\prime}=h / H$ versus Bo between hybrid substrates, for (a) $\theta_{c}^{b}=0^{\circ}$ and varying $\theta_{c}^{t}$; (b) varying $\theta_{c}^{b}$ and $\theta_{c}^{t}=0^{\circ}$.

(a)

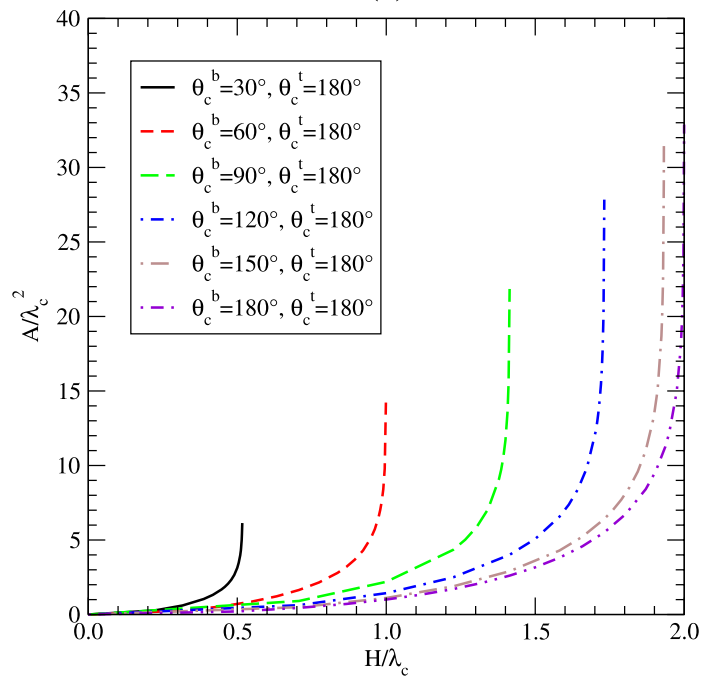

(b)

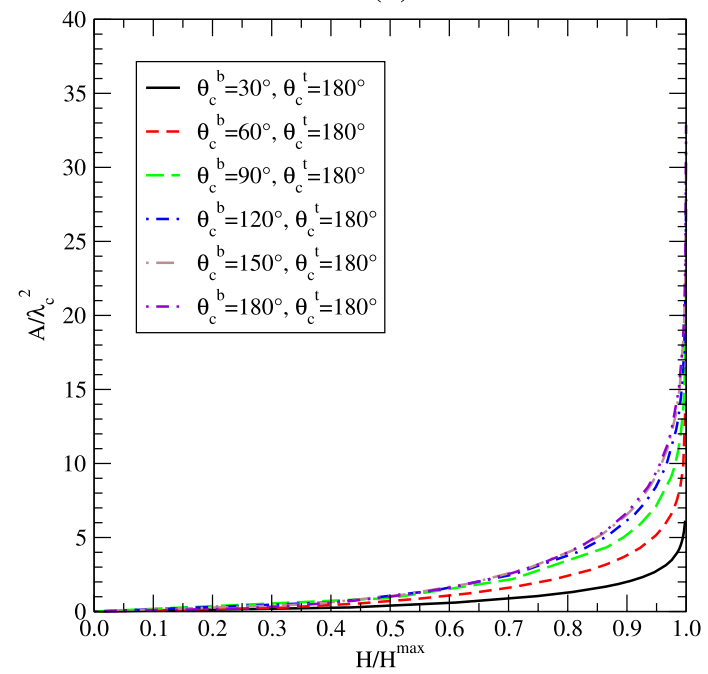

Figure 11. Dimensionless minimum cross-sectional area $A / \lambda_{c}^{2}$ of liquid bridge between hybrid substrates, for varying $\theta_{c}^{b}$ and $\theta_{c}^{t}=180^{\circ}$, versus (a) substrate separation in units of $\lambda_{c}$ (which equals $\mathrm{Bo}^{1 / 2}$ ); (b) substrate separation scaled by its maximum value.

the boundary between the top-left and bottom-left quadrants of figure 3, corresponding to a transition between one neck and zero necks. This can be seen from the fact that the bridge surfaces are vertical at the top substrate. The third panel of figure 8 is for $\theta_{c}^{b}$ and $\theta_{c}^{t}$ in the top-left quadrant of figure 3 , and therefore the bridges exhibit no necks or bulges. The fourth panel is for $\theta_{c}^{b}$ and $\theta_{c}^{t}$ on the boundary between the top-left and the top-right quadrants of figure 3 , corresponding to a transition between no bulges and one bulge (located at the bottom substrate).

In the top panel of figure 9, there is only one neck because $\theta_{c}^{b}$ and $\theta_{c}^{t}$ lie in the bottom-left quadrant of figure 3 . In the second panel, $\theta_{c}^{b}$ and $\theta_{c}^{t}$ lie on the boundary between the bottom-left and bottom-right quadrants of figure 3 , hence there is one neck (at the bottom substrate) at small Bo, or one bulge (which replaces the neck at the bottom substrate) and one neck (higher up on the bridge) at larger Bo. Note that the second neck/bulge is predicted to occur for Bo $>2$, and therefore the Bo-dependent thresholds given in the bottomright quadrant of figure 3 still apply if $\theta_{c}^{b}=90^{\circ}$, not just for $\theta_{c}^{b}>90^{\circ}$. Qualitatively similar beaviour can be seen in the fourth panel of figure 9, where again the bridge is at the transition between the bottom-left and bottom-right quadrants of figure 3. Finally, in the third and in the bottom panels of figure $9, \theta_{c}^{b}$ and $\theta_{c}^{t}$ lie in the bottom-right quadrant of figure 3 , which implies that bridges have two, one or zero necks/bulges, depending on Bo. In the third panel, the threshold separating the no necks/bulges and the one neck and one bulge regimes is $\mathrm{Bo}=8$, whereas in the bottom panel it is $\mathrm{Bo} \approx 6.78$. At these exact values of Bo, the bridges exhibit a single degenerate neck/bulge coinciding with an inflection point, as predicted previously. 
(a)

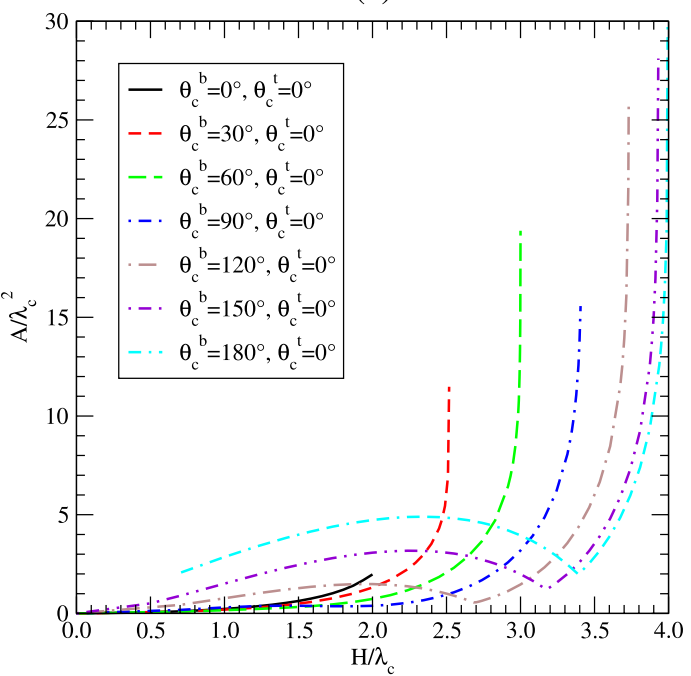

(b)

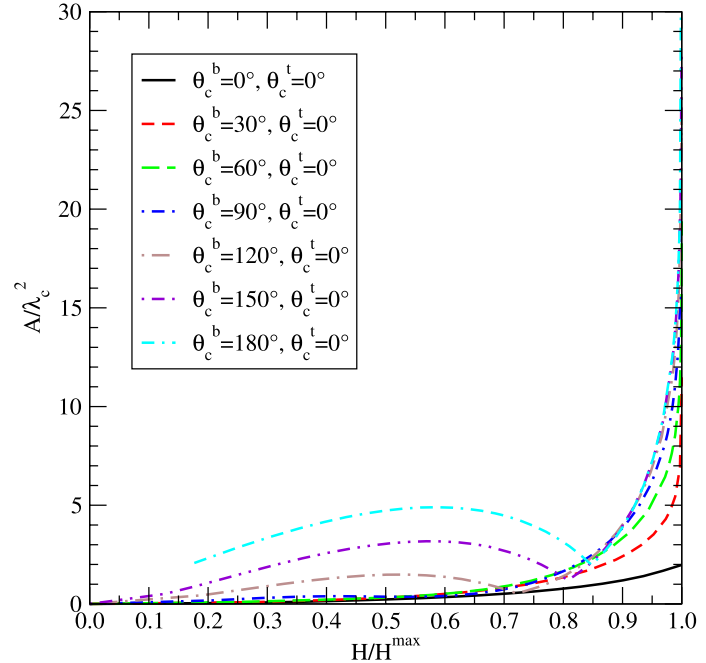

Figure 12. Dimensionless minimum cross-sectional area $A / \lambda_{c}^{2}$ of liquid bridge between hybrid substrates, for varying $\theta_{c}^{b}$ and $\theta_{c}^{t}=0^{\circ}$, versus (a) substrate separation in units of $\lambda_{c}$ (which equals $\mathrm{Bo}^{1 / 2}$ ); (b) substrate separation scaled by its maximum value.

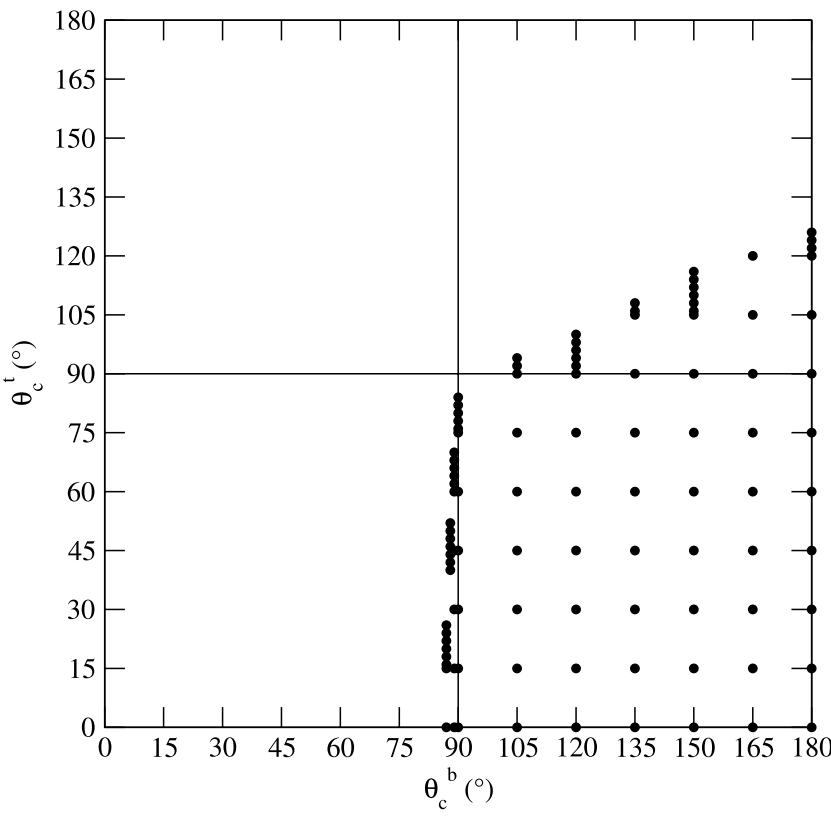

Figure 13. Regime diagram showing pairs of contact angles $\left(\theta_{c}^{b}, \theta_{c}^{t}\right)$ (black dots) for which the minimum bridge cross-sectional area is a non-monotonic function of Bo.

Figure 10 shows the vertical positions of the necks/bulges for hybrid substrates as functions of Bo for various contact angles. In figure $10(\mathrm{a})$, for $\theta_{c}^{b}=0^{\circ}$ and $\theta_{c}^{t}<90^{\circ}$, it is clear that there is only one neck, whose position on the bridge rises as either Bo or $\theta_{c}^{t}$ is increased. In figure $10(\mathrm{~b})$, for $\theta_{c}^{t}=0^{\circ}$, the situation is more complicated, with only one neck for $\theta_{c}^{b}<90^{\circ}$, whose height increases with Bo but decreases as $\theta_{c}^{b}$ is increased. For $\theta_{c}^{b}>90^{\circ}$, one neck and one bulge emerge for Bo $>2$ (as predicted in figure 3), with the neck (above) rising and the bulge (below) dropping as Bo is increased and $\theta_{c}^{b}$ is decreased.

For all $\left(\theta_{c}^{b}, \theta_{c}^{t}\right)$ except $\theta_{c}^{b}=\theta_{c}^{t}=0^{\circ}$, as Bo is increased the bridges' minimum cross-sectional area appears to diverge as Bo approaches its maximum (see figures 11 and 12). Interestingly, there is a domain of $\left(\theta_{c}^{b}, \theta_{c}^{t}\right)$ in which the minimum cross-sectional area is a non-monotonic function of Bo. As shown in figure 13, this domain coincides approximately, but not exactly, with the bottom-right quadrant of figure 3 . In both the bottomright and top-right quadrants, $x^{\prime}(0)=0$ at $\mathrm{Bo}=0$; as Bo is increased, $x^{\prime}(1)$ (the horizontal extent of the bridge at the top substrate) decreases, as more and more of the bridge's liquid content is pulled downwards by the increasing force of gravity; this is illustrated very clearly in figure 9 . These two effects combine to cause the minimum cross-sectional area to peak at some Bo and then decrease. However, if Bo goes up further, either $x^{\prime}(1)$ (in the top-right quadrant) or the $x$-position of the bridge neck (in the bottom-right quadrant) hit zero. From this point onwards, increasing Bo causes $x^{\prime}(0)$ to increase from zero: the bridge extends horizontally, close to the bottom substrate, and its minimum cross-sectional area rises steeply. A similar effect is seen in a thin sliver of the bottom-left quadrant adjacent to the bottom-right quadrant, although in this case neither $x^{\prime}(0)$ nor $x^{\prime}(1)$ become zero. Moreover, in the bottom-left quadrant the minimum cross-sectional area exhibits a true (albeit shallow) minimum as a function of Bo, whereas in the top-right and bottom-right quadrants it is actually a downward-pointing cusp where the derivative is discontinuous.

The non-monotonic nature of the minimum cross-sectional area opens up some interesting, though admittedly difficult to realise in practice, possibilities: (i) in some ranges of Bo, less liquid may be required to bridge a wider than a narrower gap between two given substrates; (ii) bi- or even tri-stable states, where the same amount of liquid suffices to bridge gaps of different widths.

\section{Conclusions}

We have integrated the Young-Laplace equation (quasi-)analytically to find the shape of the 2D liquid bridge spanning the gap between two flat, horizontal solid substrates of given 
wettabilities. We have also calculated the minimum crosssectional area of such a liquid bridge. This generalises to a physically more realistic situation our earlier work in which it was assumed that the menisci at the two substrates were connected by a liquid film of zero thickness. As shown in [1], the solution method we use yields results that are virtually indistinguishable from those obtained using the Surface Evolver, which gives us confidence that they are correct, in spite of the absence of experimental data to corroborate them.

Furthermore, we have established the range of gap widths for which the liquid bridge can exist, for given contact angles at the top and bottom substrates. In particular, we found that the absolute maximum span of a liquid bridge is four capillary lengths, for a perfectly wetting top substrate and a perfectly drying bottom substrate. If the substrates are swapped, i.e. perfectly wetting at bottom and perfectly drying at top, then no bridge can form. We have also derived the conditions for the existence, and positions of, any necks/bulges or inflection points on its surface. All these results are analytically exact and only assume that the bridge is in hydrostatic equilibrium.

However, we have not assessed the stability of the 2D bridges: this would be a whole research project in itself, which at the moment we are unsure how to perform. One possible way would be to compare the area of the surfaces bounding a 2D bridge with minimal cross-sectional area/fluid volume (which, we recall, is a liquid 'wall', or sheet, extending along the $y$-direction) with the total area of the surfaces bounding, e.g. a row of axisymmetric liquid bridges ('pillars') with the same total volume. In this sense, our calculation of the bridges of minimal cross-sectional area might be seen as a preliminary step in the assessment of film stability.

Moreover, dimensionality is known to affect the shapes of bridges in slit-pore geometry $[42,43]$. We are at the moment unable to clarify this issue, which would require a more detailed investigation.

The most significant limitation of our approach is perhaps that, besides assuming a 2D geometry, we have neglected the disjoining pressure, i.e. the direct interaction between the two bridge surfaces. This has been the subject of a number of experimental studies [44, 45] and is known to be relevant in the limit of thin bridges [46]. However, the Young-Laplace equation including disjoining pressure terms can only be solved numerically, which we defer to a later publication.

For all he above reasons, ideally we would like to be able to compare our predictions with experiments. We are not aware of any measurements on $\theta$-bridges in slit-pore geometry, although in principle this should be feasible, e.g. using a setup similar to that of [37], but where the liquid contact lines are not pinned at the substrate edges. For sufficiently long (along the $y$-direction) bridges, end effects should be negligible, thus rendering the bridge effectively $2 \mathrm{D}$.

In future work we plan to calculate the energy $E$ of a liquid bridge, which would yield the bridge-mediated force $f$ between substrates as $f=-(\mathrm{d} E / \mathrm{d} H)_{A}$. This will be repulsive for some choices of Bond number and contact angles, and attractive for others.

Finally, a few words on possible practical applications. As menstioned above, all our results have been obained for bridges in hydrostatic equilibrium:, and are therefore valid for all fluids, Newtonian or not, and of arbitrary viscosity. It is interesting to speculate that, through a judicious choice of substrate wettabilities and separations, one might be able to fabricate objects of complex cross-sectional shapes without the need for moulds or dies.

\section{Acknowledgments}

PICT acknowledges funding from the Fundação para a Ciência e Tecnologia (Portugal) through contract no. UID/ FIS/00618/2019, and thanks Instituto Politécnico de Lisboa (IPL) for travel support.

\section{Appendix. Conditions for the existence of necks/ bulges}

In order for one or two necks/bulges to exist on the liquid bridge according to equation (17), two conditions must be met: first, the discriminant under the square root must be nonnegative (so that the solutions for $h^{\prime}$ are real); second, at least one of the solutions must lie within the interval $0<h^{\prime}<1$. There will be one neck and one bulge when both solutions of equation (17) satisfy this, and only one neck/bulge if either the two solutions are identical (in which case this will be a 'degenerate' neck/bulge coinciding with an inflection point), or else if only one of the solutions satisfies this condition.

The discriminant mentioned above will be non-negative, i.e.

$$
\mathrm{Bo}^{2}+4 \mathrm{Bo}\left(\cos \theta_{c}^{b}-\cos \theta_{c}^{t}\right)+4\left(\cos \theta_{c}^{b}+\cos \theta_{c}^{t}\right)^{2} \geqslant 0
$$

either when the discriminant of equation (A.1) (which is found to be $-64 \cos \theta_{c}^{b} \cos \theta_{c}^{t}$ ) is itself negative (in which case equation (A.1) is always satisfied) or otherwise if

$$
\begin{aligned}
\text { Bo } & \leqslant 2\left(\cos \theta_{c}^{t}-\cos \theta_{c}^{b}\right)-4 \sqrt{-\cos \theta_{c}^{b} \cos \theta_{c}^{t}} \\
\text { or } \quad \text { Bo } & \geqslant 2\left(\cos \theta_{c}^{t}-\cos \theta_{c}^{b}\right)+4 \sqrt{-\cos \theta_{c}^{b} \cos \theta_{c}^{t}} .
\end{aligned}
$$

Now, it can be shown that a necessary condition for both solutions of equation (17) to satisfy $0<h^{\prime}<1$ is

$$
\text { Bo }>2\left|\cos \theta_{c}^{b}+\cos \theta_{c}^{t}\right| \quad \text { and } \quad \cos \theta_{c}^{b}<0 \quad \text { and } \quad \cos \theta_{c}^{t}>0 .
$$

Subject to these conditions (which imply that the discriminant of equation (A.1) is positive), the first inequality in equation (A.2) is automatically excluded, because $2\left|\cos \theta_{c}^{b}+\cos \theta_{c}^{t}\right|>2\left(\cos \theta_{c}^{t}-\cos \theta_{c}^{b}\right)-4 \sqrt{-\cos \theta_{c}^{b} \cos \theta_{c}^{t}}$ Hence, the condition for one neck and one bulge to exist is the second inequality in equation (A.2) together with the last two inequalities in equation (A.3), namely:

$$
\begin{aligned}
& \text { Bo }>2\left(\cos \theta_{c}^{t}-\cos \theta_{c}^{b}\right)+4 \sqrt{-\cos \theta_{c}^{b} \cos \theta_{c}^{t}} \\
& \text { and } \cos \theta_{c}^{b}<0 \text { and } \cos \theta_{c}^{t}>0 .
\end{aligned}
$$

There will be one neck/bulge if one of two conditions is satisfied. The first one (for the degenerate neck/bulge) is that the two solutions given by equation (17) coincide, which 
corresponds to replacing the inequality in equation (A.1) with an equals sign. The solutions of that equation are then given by equation (A.2), with the inequalities also replaced by equals signs. Since the first solution is again excluded by equation (A.3), which remains valid, this yields

$$
\begin{aligned}
& \text { Bo }=2\left(\cos \theta_{c}^{t}-\cos \theta_{c}^{b}\right)+4 \sqrt{-\cos \theta_{c}^{b} \cos \theta_{c}^{t}} \\
& \text { and } \cos \theta_{c}^{b}<0 \text { and } \cos \theta_{c}^{t}>0 .
\end{aligned}
$$

Alternatively, the smaller solution of equation (17) satisfies $0<h^{\prime}<1$ and the larger solution $h^{\prime}>1$, or the larger solution satisfies $0<h^{\prime}<1$ and the smaller solution $h^{\prime}<0$. It can be shown that the former case leads to the following condition:

$$
\cos \theta_{c}^{b}<0 \text { and } \cos \theta_{c}^{t}<0
$$

which corresponds to one bulge, since the contact angles satisfy $\theta_{c}^{b}>90^{\circ}$ and $\theta_{c}^{t}>90^{\circ}$, and the latter case leads to

$$
\cos \theta_{c}^{b}>0 \text { and } \cos \theta_{c}^{t}>0
$$

which corresponds to one neck, since the contact angles satisfy $\theta_{c}^{b}<90^{\circ}$ and $\theta_{c}^{t}<90^{\circ}$. Note that both of these conditions automatically satisfy equation (A.1), because its discriminant, $-64 \cos \theta_{c}^{b} \cos \theta_{c}^{t}$, is negative.

Finally, there are no necks/bulges when none of the above conditions are satisfied, i.e. when either

$$
\begin{aligned}
& \text { Bo }<2\left(\cos \theta_{c}^{t}-\cos \theta_{c}^{b}\right)+4 \sqrt{-\cos \theta_{c}^{b} \cos \theta_{c}^{t}} \\
& \text { and } \cos \theta_{c}^{b}<0 \text { and } \cos \theta_{c}^{t}>0
\end{aligned}
$$

or

$$
\cos \theta_{c}^{b}>0 \quad \text { and } \quad \cos \theta_{c}^{t}<0 .
$$

This concludes our derivation of the conditions presented in section 3 and illustrated in figure 3.

\section{ORCID iDs}

Paulo I C Teixeira (D) https://orcid.org/0000-0003-2315-2261

Miguel A C Teixeira (1) https://orcid.org/0000-0003-1205-3233

\section{References}

[1] Teixeira M A C, Arscott S, Cox S J and Teixeira P I C 2018 When is a surface foam-phobic or foam-philic? Softw. Matter 14 5369-82

[2] Pakpour M, Habib M, Møller P and Bonn D 2012 How to construct the perfect sandcastle Sci. Rep. 2549

[3] Men Y, Zhang X and Wang W 2009 Capillary liquid bridges in atomic force microscopy (AFM): formation, rupture, and hysteresis J. Chem. Phys. 131184702

[4] Edwards R B 1972 Joint tolerances in capillary copper piping joints Weld. J. 6 321-4

[5] Vagharchakian L, Restagno F and Léger L 2009 Capillary bridge formation and breakage: a test to characterize antiadhesive surfaces J. Phys. Chem. B 113 3769-75

[6] Alencar A M, Majumdar A, Hantos Z, Buldyrev S V, Stanley H E and Suki B 2005 Crackles and instabilities during lung inflation Physica A 357 18-26
[7] Persson B N J 2007 Wet adhesion with application to tree frog adhesive toe pads and tires J. Phys.: Condens. Matter 19376110

[8] Prakash M, Queré D and Bush J W M 2008 Surface tension transport of prey by feeding shorebirds: the capillary ratchet Science 320 931-4

[9] Washburn E W 1921 The dynamics of capillary flow Phys. Rev. 17 273-83

[10] Nagy N 2019 Contact angle determination on hydrophilic and superhydrophilic surfaces by using $r-\theta$-type capillary bridges Langmuir 35 5202-12

[11] Israelachvili J N 2011 Intermolecular and Surface Forces 3rd edn (Amsterdam: Elsevier)

[12] Delaunay C E 1841 Sur la surface de révolution dont la courbure moyenne est constante J. Math. Pures Appl. 6 309-14

[13] Plateau J A F 1873 Statique Expérimentale et Théorique des Liquides Soumis aux Seules Forces Moléculaires (Paris: Gauthier-Villars)

[14] Strutt J W 1878 On the instability of jets Proc. Lond. Math. Soc. 10 4-13 (Lord Rayleigh)

[15] Mason G and Clark W C 1965 Liquid bridges between spheres Chem. Eng. Sci. 20 859-66

[16] Mason G C 1970 An experimental determination of the stable length of cylindrical liquid bubbles J. Colloid Interface Sci. 32 172-6

[17] Erle M A, Gillette R D and Dyson D C 1970 Stability of interfaces of revolution with constant surface tension. The case of the catenoid Chem. Eng. J. 1 97-109

[18] Erle M A, Dyson D C and Morrow N R 1971 Liquid bridges between cylinders, in a torus, and between spheres AIChE J. 17 115-21

[19] Gillette R D and Dyson R C 1971 Stability of fluid interfaces of revolution between equal solid circular plates Chem. Eng. J. 2 44-54

[20] Padday J F 1972 Tables of the profiles of axisymmetric menisci J. Electroanal. Chem. 37 313-6

[21] Orr F M, Scriven L E and Rivas A P 1975 Pendular rings between solids: meniscus properties and capillary forces J. Fluid Mech. 67 723-42

[22] Coriell R, Hardy S C and Cordes M R 1977 Stability of liquid zones J. Colloid Interface Sci. 60 126-36

[23] Fortes M A 1982 Axisymmetric liquid bridges between parallel plates J. Colloid Interface Sci. 88 338-52

[24] Meseguer J 1984 Stability of slender, axisymmetric liquid bridges between unequal disks $J$. Cryst. Growth 67 141-3

[25] Martinez L and Perales J M 1986 Liquid bridge stability data J. Cryst. Growth 78 369-78

[26] Mazzone D N, Tardos G I and Pfeffer R 1986 The effect of gravity on the shape and strength of a liquid bridge between two spheres J. Colloid Interface Sci. 113 544-56

[27] Russo M J and Steen P H 1986 Instability of rotund capillary bridges to general disturbances, experiment and theory J. Colloid Interface Sci. 113 154-63

[28] Martinez I, Haynes J M and Langbein D 1987 Fluid statics and capillarity Fluid Sciences and Material Science in Space (Berlin: Springer) pp 53-81

[29] Vogel T I 1987 Stability of a liquid drop trapped between two parallel planes SIAM J. Appl. Math. 47 516-25

[30] Perales J M, Meseguer J and Martinez I 1991 Minimum volume stability limits for axisymmetric liquid bridges subject to steady axial acceleration J. Cryst. Growth $110855-61$

[31] Slobozhanin L A and Perales J M 1993 Stability of liquid bridges between equal disks in an axial gravity field Phys. Fluids A 5 1305-14

[32] Bezdenejnykh N A, Meseguer J and Perales J M 1992 Experimental analysis of stability limits of capillary liquid bridges Phys. Fluids A 4 677-80 
[33] van Honschoten J W, Tas N R and Elwenspoek M 2010 The profile of a capillary liquid bridge between solid surfaces Am. J. Phys. 78277

[34] Wang Y, Michielsen S and Lee H J 2013 Symmetric and asymmetric capillary bridges between a rough surface and a parallel surface Langmuir 29 11028-137

[35] Chen H, Amirfazli A and Tang T 2013 Modeling liquid bridge between surfaces with contact angle hysteresis Langmuir 29 3310-9

[36] Petkov P V and Radoev B P 2014 Statics and dynamics of capillary bridges Colloids Surf. A 460 18-27

[37] Broesch D J and Frechette J 2012 From concave to convex: capillary bridges in slit pore geometry Langmuir 28 15548-54

[38] Gao L and McCarthy T J 2009 Wetting 101 Langmuir 25 14105-15

[39] Isenberg C 1992 The Science of Soap Films and Soap Bubbles (Mineola, NY: Dover Publications)

[40] Willett C D, Adams M J, Johnson S A and Seville J P K 2000 Capillary bridges between two spherical bodies Langmuir 16 9396-405
[41] Maeda N, Israelachvili J N and Kohonen M M 2003 Evaporation and instabilities of microscopic capillary bridges Proc. Natl Acad. Sci. 100 803-8

[42] Valencia A, Brinkmann M and Lipowsky R 2001 Liquid bridges in chemically structured slit pores Langmuir 17 3390-9

[43] Swain P S and Lipowsky R 2008 Wetting between structured surfaces: liquid bridges and induced forces Europhys. Lett. 49 203-9

[44] Bergeron V 1997 Disjoining pressures and film stability of alkyltrimethylammonium bromide foam films Langmuir 13 3474-82

[45] Stubenrauch C and von Klitzing R 2003 Disjoining pressure in thin liquid foam and emulsion films-new concepts and perspectives J. Phys.: Condens. Matter 15 R1197-232

[46] Fortes M A, Teixeira P I C and Deus A M 2007 The shape of soap films and Plateau borders J. Phys.: Condens. Matter 19246106 